\title{
1-D Photonic Crystals Containing Negative Index Materials
}

\author{
J P Pandey \\ Department of Physics, M L K P G College, Balrampur (UP)
}

\begin{abstract}
Photonic crystals have been investigated by many researchers for negative refraction. These materials are typically composed of insulators and therefore, can exhibit very low losses, even at optical frequencies. Meta-materials can be strictly distinguished from other structured photonic materials. In the photonic crystals, the band-gaps arise as a result of multiple Bragg scattering in a periodic array of dielectric scatterers. The periodicity of the structure here is of the order of the wavelength and hence homogenization in this sense cannot be carried out. In meta-materials the periodicity is by comparison far less important and all the properties mainly depend on the single scatterer resonances. In this paper, the propagation of electromagnetic waves through 1-D periodic structures consisting of alternate layers of negative index material (NIM) and positive index material (PIM) is investigated. The possibility of omnidirectional reflectance and the dependence of zero- $n_{a v}$ gap on the angle of incidence and thickness scale are also studied.
\end{abstract}

\section{Introduction}

The characteristics of the photonic band gap (PBG) can be destroyed by random thickness error caused in the fabrication procedure for the conventional photonic crystal. Two new kinds of PBGs i.e. the "zero averaged refractive index" gap and "zero effective phase" gap [1,2] are proposed recently to overcome this problem. The first one is found in multilayer containing PIM and NIM whereas the second one appears in a periodic structure containing two different single-negative materials (permittivity or permeability-negative). Both these gaps are almost invariant to scale- length changes and insensitive to disorder in contrast to usual PBGs formed by the Bragg scattering. Jiang et al. [3] further demonstrated that the edge of zero- $\mathrm{n}_{\mathrm{av}}$ gap is insensitive to the incident angle for different polarizations leading to an omnidirectional gap. They also observed that defect modes inside the zero- $\mathrm{n}_{\mathrm{av}}$ gap is independent of the scaling and has weak dependence on the incident angle. The properties of layered photonic structures consisting of alternating slabs of PIM and NIM are investigated by Shadrivov et al. [4] and they demonstrated the unusual angular dependencies for the transmission of such slabs when the averaged refractive index $\left(\mathrm{n}_{\mathrm{av}}\right)$ is close to zero

The angular dependence of the band gap for the 1-D PCs consisting of alternating slabs made of PIM and NIM are studied by Daninthe et al. [5]. They found an omni gap, around the frequency where $\mathrm{n}_{\mathrm{av}}$ is zero, for a certain type of constituent left-handed medium which is independent of both angle and polarization. However, they found such a behavior for each separate polarization, by modifying the structural dimensions appropriately. Moreover, further investigation of different structures contradicts the conclusion of Jiang et al. [3]. In particular, they showed that the $\mathrm{n}_{\mathrm{av}}=0$ condition does not necessarily lead to an angle insensitive omnigap for either or both polarizations. Jiang et al (2006) [6] studied the properties of 1-D PC (with positive refractive index materials) containing an $\varepsilon$-negative and a $\mu$-negative defect which, with suitable parameters, were equivalent to a transparent material with zero effective refractive index. The special pair defect had no influence on spectral gap formed by interference of propagating waves in positive refractive index materials. However, the field distribution were modified, especially gap edge field could be highly localized.

The variance of a zero- $\phi_{\text {eff }}$ gap with an arbitrary incident angle and polarization were also investigated by Jiang et al. (2005) [7]. They found that a zero- $\phi_{\text {eff }}$ gap can be an omnidirectional gap (OBG) whose edges are insensitive to incident angle and polarization. In contrast to OBG from a zero- $n_{\mathrm{av}} \mathrm{gap}$, it can be widened by varying the ratio of the thicknesses of two media and is independent of polarization under special conditions. A zero- $n_{\text {av }}$ gap was also found in photonic spectra of Fibonacci quasi-periodic structure consisting of alternating slabs of positive and negative refractive index material by $\mathrm{Li}$ et al. [8]. Zhang et al. [9] demonstrated that multiple-channeled filtering can be realized by applying heterostructures consisting of two kinds of singlenegative materials inserted with defects.

New effects are produced by the interaction between two kinds of non-Bragg gaps that can coexist in PCs which combine ordinary materials and dispersive metamaterials [10-15]. This effect is produced by the unavoidable (and usually strong) dispersive character of metamaterials.

In this communication, omnidirectional reflection is theoretically investigated from the multilayer structure with alternate layers of PIM and NIM as its constituent layers. 


\section{Mathematical Formulation:}

Consider the wave propagation in a periodic multilayer of positive and negative index materials of parameters $\left(\varepsilon_{1}, \mu_{1}\right)$ and $\left(\varepsilon_{2}, \mu_{2}\right)$ with $\mathrm{d}_{1}$ and $\mathrm{d}_{2}$ as the width of the two constituent layers. $\mathrm{d}=\mathrm{d}_{1}+\mathrm{d}_{2}$ is the period of the unit cell. The corresponding refractive index is given by $\mathrm{n}_{\mathrm{j}}= \pm \sqrt{\varepsilon_{\mathrm{j}}} \sqrt{\mu_{\mathrm{j}}}$ (negative sign for negative index material). We consider an oblique propagation of monochromatic electromagnetic field (with time dependence $\mathrm{e}^{-\mathrm{i} \omega t}$ ) in a periodic structure with oblique wave vector $\beta$ along the $\mathrm{z}$-axis. The electric field vector of a general plane wave solution of the Helmholtz equation can be written in the form: $\mathrm{E}(\mathrm{x}, \mathrm{t})=\mathrm{E}(\mathrm{x})$

$\exp [-\mathrm{i}(\omega \mathrm{t}+\beta \mathrm{y})]$ (1) where $\beta=\frac{\omega}{\mathrm{c}} \mathrm{n}_{\mathrm{j}} \cos \theta_{\mathrm{j}}$, is the $\mathrm{y}$ component of the propagation vector, and $\mathrm{E}(\mathrm{x})$ is the sum of incident wave and reflected wave at the interfaces. The magnetic field component can be obtained from

$$
\begin{aligned}
& \overrightarrow{\mathrm{H}}=\frac{\mathrm{i}}{\omega \mu(\mathrm{x})} \vec{\nabla} \times \overrightarrow{\mathrm{E}} \\
& \mathrm{E}_{1 \mathrm{z}}=\mathrm{e}^{\mathrm{i} \beta \mathrm{y}}\left[\mathrm{Ae}^{\mathrm{i} \mathrm{k}_{1 \mathrm{x}} \mathrm{x}}+\mathrm{Be}^{-\mathrm{i} \mathrm{k}_{1 \mathrm{x}} \mathrm{x}}\right] \quad \mathrm{H}_{1 \mathrm{y}}=-\frac{\mathrm{k}_{1 \mathrm{x}}}{\omega \mu_{1}} \mathrm{e}^{\mathrm{i} \beta \mathrm{y}}\left[\mathrm{Ae}^{\mathrm{ik_{1x } \mathrm { x }}}-\mathrm{Be}^{-\mathrm{i} \mathrm{k}_{1 \mathrm{x}} \mathrm{x}}\right] \\
& \mathrm{H}_{1 \mathrm{x}}=-\frac{\beta}{\omega \mu_{1}} \mathrm{e}^{\mathrm{i} \beta \mathrm{y}}\left[\mathrm{Ae}^{\mathrm{i} \mathrm{k}_{1 \mathrm{x}} \mathrm{x}}+\mathrm{Be}^{-\mathrm{ik}_{1 \mathrm{x}} \mathrm{x}}\right] \quad \text { in region } \mathrm{I} \\
& \mathrm{E}_{2 \mathrm{z}}=\mathrm{e}^{\mathrm{i} \beta \mathrm{y}}\left[\mathrm{Ce}^{\mathrm{i} \mathrm{k}_{2 \mathrm{x}} \mathrm{x}}+\mathrm{D} \mathrm{e}^{-\mathrm{i} \mathrm{k}_{2 \mathrm{x}} \mathrm{x}}\right] \quad \mathrm{H}_{2 \mathrm{y}}=-\frac{\mathrm{k}_{2 \mathrm{x}}}{\omega \mu_{2}} \mathrm{e}^{\mathrm{i} \beta \mathrm{y}}\left[\mathrm{Ce}^{\mathrm{i} \mathrm{k}_{2 \mathrm{x}} \mathrm{x}}-\mathrm{D} \mathrm{e}^{-\mathrm{ik} \mathrm{k}_{2 \mathrm{x}} \mathrm{x}}\right] \\
& \mathrm{H}_{2 \mathrm{x}}=-\frac{\beta}{\omega \mu_{2}} \mathrm{e}^{\mathrm{i} \beta \mathrm{y}}\left[\mathrm{Ae}^{\mathrm{i} \mathrm{i}_{2 \mathrm{x}} \mathrm{x}}+\mathrm{Be}^{-\mathrm{i} \mathrm{k}_{2 \mathrm{x}} \mathrm{x}}\right] \quad \text { in region II }
\end{aligned}
$$

where $\mathrm{k}_{\mathrm{j}}$ is the component of the wave vector along $\mathrm{x}$-axis in region $\mathrm{j}=1,2$ i.e.

$$
\mathrm{k}_{\mathrm{j}}^{2}=\varepsilon_{\mathrm{j}} \mu_{\mathrm{j}} \frac{\omega^{2}}{\mathrm{c}^{2}}-\beta^{2}
$$

Here, $\mathrm{c}$ is the speed of light in vacuum and $\beta$ is the $\mathrm{y}$-component of wave vector. Imposing the periodicity constant $\mathrm{E}[(\mathrm{x}+\mathrm{d}), \mathrm{y}]=\mathrm{E}(\mathrm{x}, 0) \exp (\mathrm{i} \mathrm{K} \mathrm{d}+\mathrm{i} \beta \mathrm{y})$, where $\mathrm{d}=\mathrm{d}_{1}+\mathrm{d}_{2}$ is the periodic thickness of unit cell. $\mathrm{K}$ is the dimensionless Bloch wave vector of the periodic unit cell, which define the transmission across the layer and its dependence on the wave vector component along the $\beta$ can be found explicitly for two-layer periodic structure [16]. The tangential component of electric and magnetic fields should be continuous at $\mathrm{x}=0$ and $\mathrm{x}=\mathrm{d}_{2}$ i.e.

$$
\mathrm{E}_{1 \mathrm{z}}\left(\mathrm{x}=0^{-}\right)=\mathrm{E}_{2 \mathrm{z}}\left(\mathrm{x}=0^{+}\right) \quad(5 \mathrm{a}) \quad \mathrm{H}_{1 \mathrm{y}}\left(\mathrm{x}=0^{-}\right)=\mathrm{H}_{2 \mathrm{y}}\left(\mathrm{x}=0^{+}\right)(5 \mathrm{~b})
$$

In 1-D periodic structure, the propagating waves have the form of Bloch modes, for which the electric field amplitude satisfy the periodicity condition.

$$
\mathrm{E}_{2 z}\left(\mathrm{x}=\mathrm{d}_{2}\right)=\mathrm{E}_{1 \mathrm{z}}\left(\mathrm{x}=-\mathrm{d}_{1}\right) \mathrm{e}^{\mathrm{iKd}+\mathrm{i} \beta \mathrm{y}}(6 \mathrm{a}) \quad \mathrm{H}_{2 \mathrm{y}}\left(\mathrm{x}=\mathrm{d}_{2}\right)=\mathrm{H}_{1 \mathrm{y}}\left(\mathrm{x}=-\mathrm{d}_{1}\right) \mathrm{e}^{\mathrm{i} K \mathrm{~d}+\mathrm{i} \beta \mathrm{y}}(6 \mathrm{~b})
$$

where $d=d_{1}+d_{2}$ is the period of the structure. We obtain the matrix M characterizing the wave scattering in the periodic structure for TE mode,

$$
\begin{aligned}
& \left(\begin{array}{l}
A_{n-1} \\
B_{n-1}
\end{array}\right)=M\left(\begin{array}{l}
A_{n} \\
B_{n}
\end{array}\right) \quad \text { (7) where } \quad M=\left(\begin{array}{ll}
M_{11} & M_{12} \\
M_{21} & M_{22}
\end{array}\right) \text { with } \\
& M_{11}=e^{i k_{1 x} d_{1}}\left[\cos \left(k_{2 x} d_{2}\right)+\frac{i}{2}\left(\frac{k_{2 x} \mu_{1}}{k_{1 x} \mu_{2}}+\frac{k_{1 x} \mu_{2}}{k_{2 x} \mu_{1}}\right) \sin \left(k_{2 x} d_{2}\right)\right] \\
& \mathrm{M}_{12}=\mathrm{e}^{-\mathrm{ik}_{1 \mathrm{x}} \mathrm{d}_{1}}\left[\frac{\mathrm{i}}{2}\left(\frac{\mathrm{k}_{2 \mathrm{x}} \mu_{1}}{\mathrm{k}_{1 \mathrm{x}} \mu_{2}}-\frac{\mathrm{k}_{1 \mathrm{x}} \mu_{2}}{\mathrm{k}_{2 \mathrm{x}} \mu_{1}}\right) \sin \left(\mathrm{k}_{2 \mathrm{x}} \mathrm{d}_{2}\right)\right] \quad \mathrm{M}_{21}=\overline{\mathbf{M}}_{12} \quad \text { and } \mathrm{M}_{22}=\overline{\mathbf{M}}_{11}
\end{aligned}
$$

The characteristic matrix can be related easily by the plane wave amplitudes of the left, right and back waves in terms of transmission ( $t$ ) and reflection (r) coefficients for $\mathrm{N}^{\text {th }}$ period as, 


$$
\left(\begin{array}{l}
\mathrm{t} \\
0
\end{array}\right)=\mathrm{M}\left(\begin{array}{l}
1 \\
\mathrm{r}
\end{array}\right)
$$

where, $\quad \mathbf{M}_{11}=\mathrm{M}_{11} \mathrm{U}_{\mathrm{N}-1}-\mathrm{U}_{\mathrm{N}-2}, \quad \mathbf{M}_{12}=\mathrm{M}_{12} \mathrm{U}_{\mathrm{N}-1,}, \quad \mathbf{M}_{22}=\mathrm{M}_{22} \mathrm{U}_{\mathrm{N}-1}-\mathrm{U}_{\mathrm{N}-2}$,

and, $\quad \mathrm{U}_{\mathrm{N}}=\sin [(\mathrm{N}+1) \mathrm{K}(\omega) \mathrm{d}] / \sin [\mathrm{K}(\omega) \mathrm{d}]$

So, that the reflection and transmission coefficient $r$ and $t$ are given by,

$$
r=-\frac{M_{21}}{M_{22}} \quad \text { and } \quad t=M_{11}-\frac{M_{12} M_{21}}{M_{22}}
$$

Hence, the reflectance and transmittance are given as,

$$
\mathrm{R}=|\mathrm{r}|^{2} \quad \text { and } \quad \mathrm{T}=|\mathrm{t}|^{2}
$$

\section{Results And Discussion:}

More robust zero- $\mathrm{n}_{\mathrm{av}}$ gap can be obtained by lowering the zero- $\mathrm{n}_{\mathrm{av}}$ frequency, because a lower frequency i.e. a longer wavelength means a smaller phase shift when a wave propagates through a medium. Since a smaller frequency shift is required to compensate the smaller phase shift, so the low frequency zero- $\mathrm{n}_{\mathrm{av}}$ gap should depend a little on the incident angle. For this, a negative index material is used with effective permittivity and permeability following plasma like dispersion as given by Jiang et al. [6]:

$$
\varepsilon(\omega)=1.21-\frac{100}{\omega^{2}}, \quad(11) \quad \text { and, } \quad \mu(\omega)=1-\frac{100}{\omega^{2}}
$$

where $\omega$ is in GHz. The 1D periodic multilayer consists of alternating layers of (NIM) with permittivity and permeability as above and a (PIM) with $\mathrm{n}_{\mathrm{P}}=3\left(\varepsilon=9\right.$ and $\mu=1$ ). The thickness of the two layers are $\mathrm{d}_{\mathrm{N}}=6$ $\mathrm{mm}$ and $\mathrm{d}_{\mathrm{P}}=12 \mathrm{~mm}$. Variation of permittivity (solid line) and permeability (dotted line) with frequency of the NIM is given in figure 1 (a). The interest of the frequency region is in which $\varepsilon(\omega)$ and $\mu(\omega)$ are simultaneously negative. The plot of refractive index for NIM (solid line represents real part and dotted line imaginary part) is shown in figure 1 (b). Average refractive index $n_{\mathrm{av}}$ of the system of NIM and PIM is equal to zero at $0.6 \mathrm{GHz}$ (figures 2(a)). The zero- $\mathrm{n}_{\text {av }}$ gap is formed around this point (figures 2(b)-(c)). The second gap which appears in the region where $\varepsilon(\omega)$ and $\mu(\omega)$ are both positive is a Bragg gap because in this region, layer $\mathrm{N}$ is an ordinary material with positive refractive index.

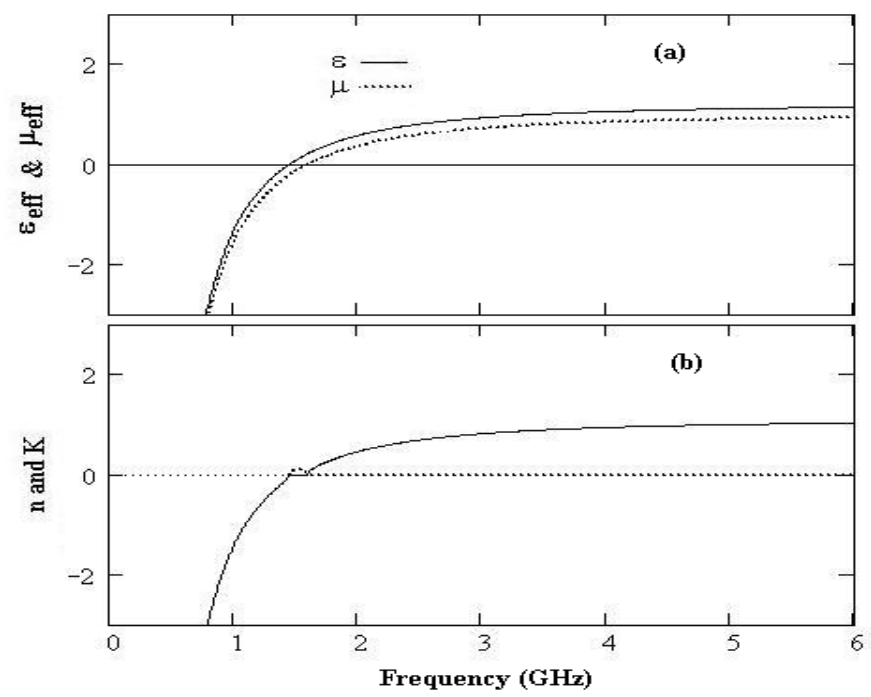

Figure 1 Optical constants for the NIM (a) Permittivity (solid line) and permeability (dotted line) versus frequency. (b) Index of refraction versus frequency (solid curve is real component and dotted curve is imaginary component).

In order to observe the influence of the variation of angle of incidence, the transmittance spectra of the system is plotted for TE and TM polarizations at different angles of incidence (figure 3). There is nearly no change in zero- $\mathrm{n}_{\mathrm{av}}$ gap and is insensitive to the change in angle of incidence for both TE and TM polarizations. So, the zero- $\mathrm{n}_{\mathrm{av}}$ gap is an omnidirectional gap for both the polarizations. Now, the transmission versus frequency is plotted for three scaled thicknesses for both TE and TM polarizations at normal incidence (figure 4) 
to see the dependence of transmission spectra on the thickness scale. The scaled thickness ratios are $d_{N}: d_{P}=6$ mm:12 mm, $9 \mathrm{~mm}: 18 \mathrm{~mm}$ and $12 \mathrm{~mm}: 24 \mathrm{~mm}$. It is clear that when lattice constant increases, the zero- $\mathrm{n}_{\mathrm{av}}$ gap does not change with scaling and remains unchanged. However, the Bragg gap are scale sensitive and they shift downwards in frequency.

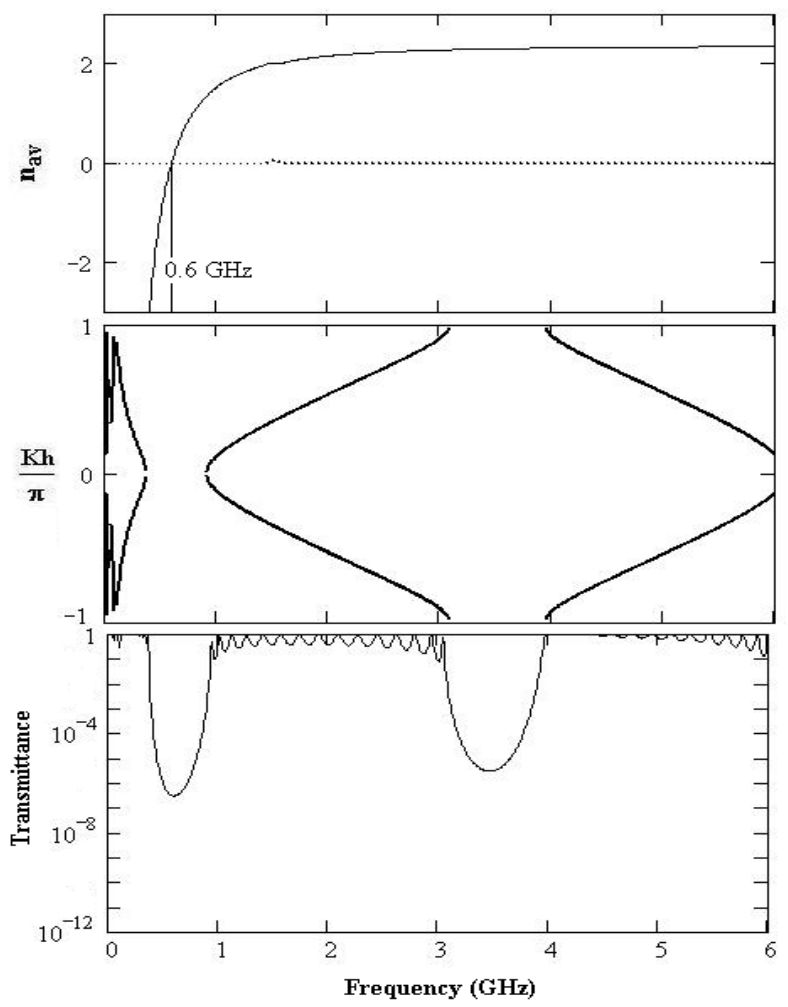

Figure 2 (a) Average refractive index versus frequency $n_{a v}=0$ at $0.6 \mathrm{GHz}$. (b) Dispersion relation for the system showing a bandgap centered around $0.6 \mathrm{GHz}$. (c) Transmission versus frequency corresponding to the band structure in (b)

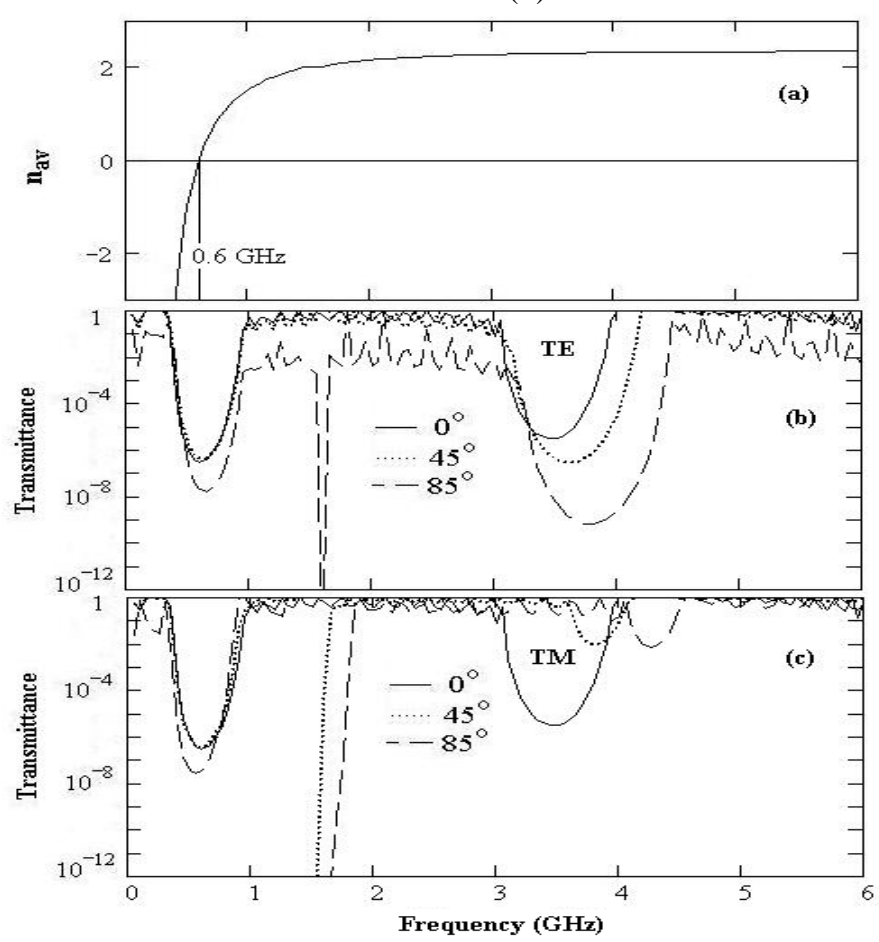

Figure 3 (a) $n_{a v}$ versus frequency showing $n_{a v}=0.6 \mathrm{GHz}$. Transmittance of the photonic crystal with 16-pairs of alternating layers of NIM (thickness $=6 \mathrm{~mm}$ ) and air $(\mathrm{PIM})($ thickness $=12 \mathrm{~mm}$ ) at different incident angles for (b) TE polarization and (c) TM polarization. 


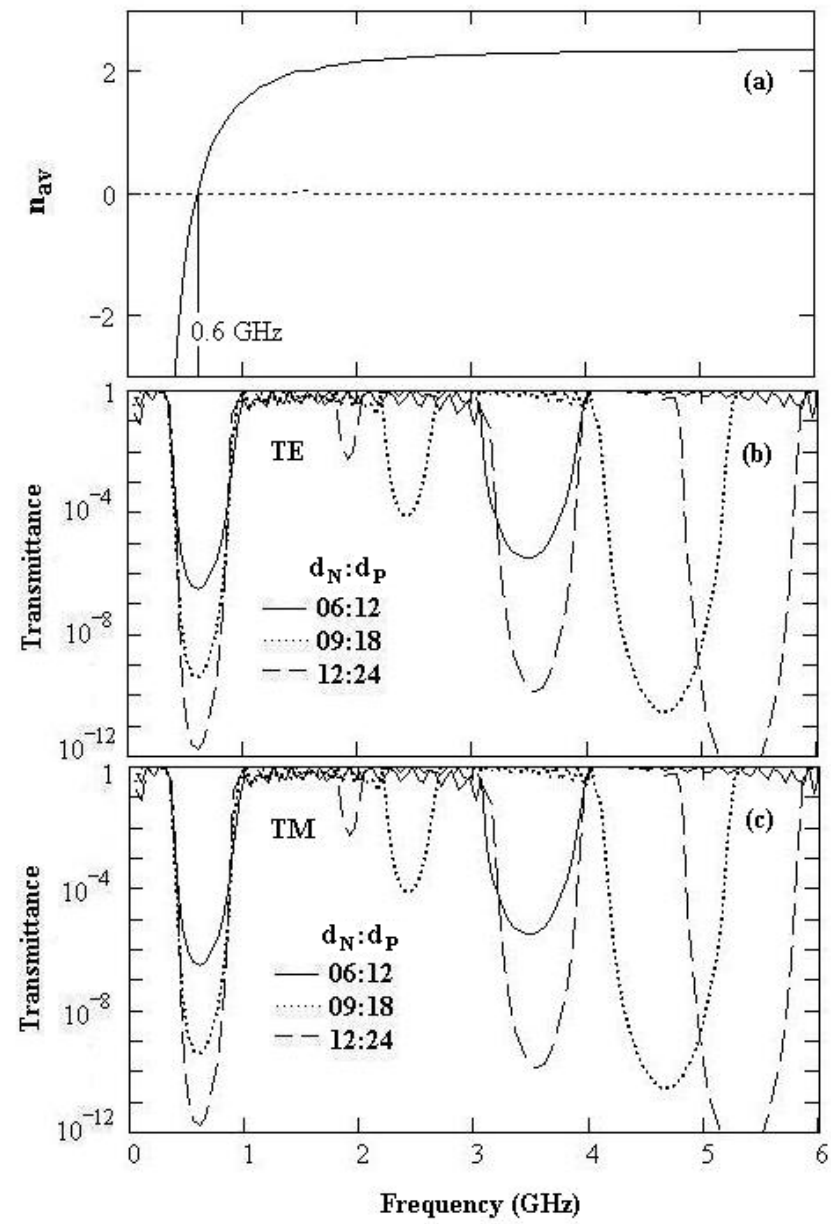

Figure 4 (a) $n_{a v}$ versus frequency plot shows the $n_{a v}=0$ at $0.6 \mathrm{GHz}$. The variation of transmittance spectra with the thickness scales under normal incidence for (b) TE polarization and (c) TM polarization. Three ratios of thickness are $d_{N}: d_{P}=6: 12 \mathrm{~mm}, 9: 18 \mathrm{~mm}$, and 12:24 mm.

\section{References}

[1]. J. Li, L. Zhou, C. T. Chan, and P. Sheng, Phys. Rev. Lett. 90, 083901, 2003.

[2]. H. Jiang, H. Chen, H. Li, Y. Zhang, J. Zi, and S. Zhu, Phys. Rev. E 69, 066607, 2004.

[3]. H. Jiang, H. Chen, H. Li, Y. Zhang, and S. Zhu, Appl. Phys. Lett. 83, 5386-5388, 2003.

[4]. I. V. Shadrivov, A. A. Sukhorukov, and Y. S. Kivshar, Appl. Phys. Lett. 82, 3820-3822, 2003

[5]. H. Daninthe, S. Foteinopoulou, C. M. Soukoulis, Photonics and Nanostructures-Fundamentals and Applications 4, 123-131, 2006

[6]. H. Jiang, H. Chen and S. Zhu, Phy. Rev. E 73, 046601, 2006.

[7]. J. Hai-Tao, C. Hong, Li H-Q and Z. Ye-Wen, Chin. Phys. Lett. 22, 884-886, 2005.

[8]. J. Li, D. Zhao, Z. Liu, Physics Letters A 332, 461- 468, 2004.

[9]. H. Y. Zhang, Y. P. Zhang, P. Wang, and J. Q. Yao, Appl. Phys. Lett. 101, 013111, 2007

[10]. J. A. Monsoriu, R. A. Depine, and M. L. Mart'ýnez-Ricci, Optics Express 14, 12958-67, 2006

[11]. R. A. Depine, M. L. Martínez-Ricci, J. A. Monsoriu, E. Silvestre and P. Andrés, Physics Letters A 364, 352-355, 2007.

[12]. S. K. Singh, J. P. Pandey, K. B. Thapa and S. P. Ojha, Solid State Communications doi: 10.1016, j.ssc.2007.05.016, 2007.

[13]. C. Sabah and H. G. Roskos, Progress In Electromagnetics Research, Vol. 124, 301-314, 2012.

[14]. G. N. Pandey, khem B Thapa and S. P. Ojha, Optik - International Journal for Light and Electron Optics, Volume 125, 252-256. 2014.

[15]. J P Pandey, IOSR Journal of Applied Physics (IOSR-JAP), Volume 9, Issue 2, pp 59-63 Ver. I (Mar. - Apr. 2017).

[16]. P. Yeh, Optical waves in layered media (Willey, New York, 1988). 